\section{P32 NO GUT SYNDROME IN PAEDIATRIC PATIENTS}

Elena Cernat, Lilianne Gomez Lopez, Veena Zamvar, Dinesh Rawat, lan Sugarman. Leeds Teaching Hospitals NHS Trust

10.1136/flgastro-2021-bspghan.41

Introduction/Background No gut syndrome or near total enterectomy (NTE) is defined as the removal of the entire jejunum and ileum, a rarely performed procedure indicated in very specific situations and associated with parenteral nutrition (PN) dependency, multiple complications and poor quality of life in general. To date, only few cases have been reported in literature, all in the adult setting.

Aim The aim of this report is to raise awareness of this condition in paediatric patients and the difficulties encountered in their management.

Subjects and Methods Two male patients (2 and 5 year old) are presented. One secondary to an ischaemic event of unknown cause and the second post a failed small bowel transplant, both suffering complications specific to this condition.

Results Case 1: First-born to non-consanguineous parents at 37 weeks of gestation with 22 q11 deletion. Born with large VSD, had several respiratory infections initially. At 2.5 months while in PICU with a respiratory deterioration, developed intestinal infarction from duodenum to sigmoid colon and was left with D1-D2 (D2 showed patches of ischemia). A duodenal tube was left in the duodenal stump and was started on PN. A month later, a contrast study showed 2 fistulas between the duodenum and the rectum that resolved spontaneously. Currently he continues to be total PN dependent having only some water orally (thicker consistency can block the tube), has a balloon $\mathrm{G} / \mathrm{J}$ tube pushed into the stomach due to a narrowed duodenal stump to try and minimise vomiting, and has issues with leakage around the tube. His liver function tests (LFTs) have been abnormal intermittently and his liver US shows hepatomegaly and gallstones.

Case 2: Third child to non-consanguineous parents born at 35 weeks of gestation with complex gastroschisis, jejunal atresia and hypoplastic colon - remaining anatomy $15 \mathrm{~cm}$ from GJ junction. Initial treatment involved placement/ removal of a silo followed by bowel expansion, formation of a jejunostomy and a mucous fistula closed a year later when he had a jejunocolonic anastomosis. He had several interventions but was unable to tolerate minimal enteral amounts with a high stoma output despite treatment with octreotide. He underwent an isolated bowel transplant at 4 years of age followed by severe rejection not responsive to ATG/Campath and complicated with PTLD. He had a graft enterectomy with remaining anatomy $5 \mathrm{~cm}$ of duodenum and leakage from the duodenal stump. Currently he has a mucus fistula, an abdominal drain and a gastrostomy. Type 1 Chiari malformation was incidentally found and he developed seizures. He has intestinal failure associated liver disease and renal stones. He continues to have frequent admissions due to his health needs and frequent complications.

Summary and Conclusion Advances in PN have made possible the long-term survival of patients with no gut syndrome in specialized centres but complications, patient and family quality of life, use of health resources, medical and surgical challenges and overall outcomes are important aspects to be considered and more paediatric data is necessary.

\section{P33 OUTCOME OF CENTRAL VENOUS CATHETER REPAIR IN CHILDREN WITH INTESTINAL FAILURE}

Zafar Zaidi, Rhona Shepherd, Hannah Littlechild, Susan Hill, Jutta Koeglmeier. Great Ormond Street Hospital

\subsection{6/flgastro-2021-bspghan.42}

Introduction Children with intestinal failure (IF) requiring a central venous catheter (CVC) for long term parenteral nutrition $(\mathrm{PN})$ are at risk of CVC breakage and infection. Modern IF management aims to preserve vascular access sites. CVC repair rather than removal is hence carried out for broken catheters when possible. Data suggesting an increased risk for central line-associated bloodstream infections (CLABSIs) associated with CVC repair are limited. The aim of this study was to describe outcomes of CVC repairs among a cohort of children with IF dependant on home PN and risk factors leading to catheter repair.

Material and Methods All paediatric patients (ages 0-17 years) with CVC dependency enrolled in the IF rehabilitation program of a large tertiary referral centre who underwent a CVC repair between January 2019 and November 2020 were included in the study. Data were collected retrospectively from the clinic notes. Risk factors associated with catheter breakage and incidence of CLABSIs post repair were documented. Descriptive statistics including medians, percentages and frequencies were used.

Results Forty children, 15 males (37\%) and 25 females (63\%), received $\mathrm{PN}$ during the 2-year study period. 15/40 $(37.5 \%$ ) patients, 8 girls $(53 \%)$ and age ranging from 1 to 17 years underwent a total of 29 CVC repairs (mean 0.36 repairs per patient per year). The highest number of repairs occurred in patients under 5 years of age $(n=8 / 15 ; 53 \%$; $33 \%$ females). Around half of the patients $53.3 \% \quad(n=8 / 15)$ underwent $>2$ repairs including one patient with 3 and another with 5 repairs. Median time between two repairs was 6 months. The most common reason for repair was CVC fracture caused by biting (41\%) followed by repair for total catheter occlusion with intraluminal PN deposition (13.2\%), while $6.8 \%$ repairs were done for wear \& tear, thromboembolic occlusion, mechanical trauma, increased pressure and weak catheter (one each). Repair was successful in $100 \%$ cases with none requiring CVC replacement. Blood cultures (BC) taken post CVC repair were negative in the majority of cases $(27 / 29 ; 93 \%)$. One child had a positive CVC culture taken pre repair in the referring hospital but negative BC post repair making contamination leading to a false positive result likely. Only one patient had a confirmed CLABSI post repair. However, this child presented late 3 days after the initial catheter breakage and catheter salvage was successful with antibiotic therapy.

Conclusion In our cohort of home PN dependant IF patients infection rate after CVC repair was minimal. CVC repair rather than removal is recommended to preserve central venous access sites and reduce the need for general anaesthesia. Support from a central vascular access team skilled in catheter repair is essential. 